\title{
Non-Epitheliotropic Cutaneous Lymphoma with Systemic Dissemination in a Dog
}

\author{
Laís Guedes Rosseto', Beatriz Crepaldi Aléssio Pitol', Paulo Antonio Terrabuio Andreussi', \\ Mariana Isa Poci Palumbo' ${ }^{\text {, Adriana Ventura2 }}{ }^{2}$ Veronica Jorge Babo-Terra' ${ }^{1}$
}

\begin{abstract}
Background: Cutaneous lymphoma is a highly malignant neoplasm, which can originate in the epidermis or dermis, as well as be disseminated to other organs such as lung, heart, arm, liver and bone marrow. It comes in the form of nodes of various sizes, erythematous and alopecic, itching may or may not occur. The diagnosis is made by cytological and histopathological examination of the compromised tissue. However, it is important to perform complementary tests for clinical staging and prognostic characterization. The objective is to report a case of non-epitheliotropic cutaneous lymphoma with systemic dissemination in a dog.

Case: A female mixed bred adult canine was attended at the Veterinary Hospital of the Federal University of Mato Grosso do Sul with a history of progressive weight loss and the presence of alopecic and non-pruritic subcutaneous nodules in the torso, nasal plane and pelvic limbs, starting 4 months ago. After approximately 20 days, the presence of rapidly evolving ulcerated nodules was noted. On physical examination, generalized lymphadenomegaly was observed and among the dermatological findings were multiple nodules of varying sizes with the presence of ulceration in the center of the lesions, alopecia, erythema and raised edges, in the region of the nasal sinus, pelvic and thoracic limbs, tail, thoracolumbar and abdominal region. The animal also presented right pelvic limb edema with painful sensibility to manipulation CBC and biochemical tests (albumin, alanine aminotransferase, creatinine, urea, alkaline phosphatase, globulins, total proteins and fractions) were performed, being observed as normocytic normochromic type anemia alteration (erythrocytes: 2.78 106/ $\mu \mathrm{L}$; hemoglobin: $6.8 \mathrm{~g} / \mu \mathrm{L}$; globular volume: $18.8 \%)$, leukopenia $\left(4,000 / \mathrm{mm}^{3}\right)$ with presence of metamyelocytes $(120 /$ $\left.\mathrm{mm}^{3}\right)$ and rods $\left(1,080 / \mathrm{mm}^{3}\right)$ and lymphopenia $\left(80 / \mathrm{mm}^{3}\right)$. Three samples of the nodules were collected for histopathological examination and a definitive diagnosis of cutaneous lymphoma was obtained. The material was then submitted to immunohistochemical examination, which showed that it was a non-epitheliotropic cutaneous lymphoma of $\mathrm{T}$ immunophenotype. Due to the compromised quality of life and unfavorable prognosis, the owner opted for euthanasia of the animal. In the necropsy examination, lymph nodes, subcutaneous tissue, skeletal muscle, heart, pericardial sac, tongue and multifocal infiltrate of neoplastic cells were observed, findings suggestive of multicentric lymphoma or infiltrations by dissemination of cutaneous lymphoma.

Discussion: Non-epitheliotropic skin lymphomas exhibit rapid progression and infiltration into lymph nodes and subsequent systemic involvement. The diagnosis is based on clinical-dermatological signs, fine needle cytology, histopathological and immunohistochemical examination. Normocytic normochromic anemia is the most observed alteration in patients with lymphoma, followed by leukocytosis, leukopenia, thrombocytopenia, neutrophilia and leukoerythroblastic reactions. Dogs with T-cell lymphoma have a worse prognosis for life span and disease-free intervals than those with B-cell lymphoma, so immunophenotyping is critical to determine prognosis. In the present report, histopathological and immunohistochemical examinations were decisive for the diagnosis of the present report, because the expression CD3 and the negativity for CD79a proved that the neoplasm is of T lymphocyte lineage. The definitive diagnosis was obtained by histopathology, however, immunohistochemistry determined the immunophenotype of the neoplasia as non-epitheliotropic T lymphocyte.
\end{abstract}

Keywords: dermatology, lymphoma, malignancy, nodules, oncology. 


\section{INTRODUCTION}

Cutaneous lymphoma is considered a rare form of lymphoma in dogs, representing only $1 \%$ of cutaneous neoplasms [4]. It may involve the oral mucosa and other organs, such as lymph nodes, spleen, liver and bone marrow [6]. Histologically, it can be classified as epitheliotropic, in which it has tropism by the epidermis, or not epitheliotropic, which is located only in the dermis [7].

Four stages are described for characterization of epitheliotropic lymphoma or mycosis fungoides. First, the generalized form, with presence of erythema and peeling; the second stage, mucocutaneous erythema, with infiltration, depigmentation and ulceration; the third stage consists of solitary cutaneous plaque, multiple or nodules and in the fourth stage occurs the infiltration, ulceration in oral mucosa and finally the dissemination with nodal involvement and occasionally, circulating Sezáry cells [3].

The clinical signs are varied and depend on the anatomical classification and extent of the disease. The clinical presentation is non-specific and can mimic many dermatitis, and may be accompanied by non-specific signs such as hyperoxia and weight loss [8]. The diagnosis is made by cytological and histopathological examination; however, it is important to perform complementary exams for clinical staging and prognostic characterization [6]. This study aims to report a case of non-epitheliotropic cutaneous lymphoma with systemic dissemination in a dog.

\section{CASE}

A female mixed bred adult dog, with a history of progressive weight loss and presence of alopecic and non-pruritic subcutaneous nodules in the torso, nasal plane and pelvic limbs, starting 4 months ago, was treated at the Veterinary Hospital of the Federal University of Mato Grosso do Sul (FAMEZ) in Campo Grande, Mato Grosso do Sul. About 20 days ago, the presence of rapidly evolving ulcerated nodules had been noted. The animal had already been attended by another veterinary doctor who did not perform complementary exams and prescribed topical healing drugs, without success. On physical examination, generalized lymphadenomegaly was observed, with no other changes. On dermatological examination, multiple nodules of varying sizes were observed with the presence of ulceration in the center of the lesions, alopecia, erythema and elevated edges, in the region of the nasal sinus, pelvic and thoracic limbs, tail, thoracolumbar and abdominal regions. The animal presented right pelvic limb edema with painful sensitivity to manipulation (Figure 1A).

$\mathrm{CBC}$ and serum albumin, alanine aminotransferase, creatinine, urea, alkaline phosphatase, globulins, total proteins and fractions were requested. A fine needle aspiration puncture of the nodules was performed for cytology, hemogasometry for calcium ion dosage, chest radiography and abdominal ultrasonography. The CBC showed normocytic normochromic anemia (erythrocytes: $2.78106 / \mu \mathrm{L}$, reference: 5.5 to $8.5106 / \mu \mathrm{L}$; hemoglobin: $6.8 \mathrm{~g} / \mu \mathrm{L}$, reference: 12 to $18 \mathrm{~g} / \mu \mathrm{L}$; globular volume: $18.8 \%$, reference: 37 to 55 $\%$, leukocyte count: $4.000 / \mathrm{mm}^{3}$, reference: 6.000 to $\left.17.000 / \mathrm{mm}^{3}\right)$, presence of metamyelocytes $\left(120 / \mathrm{mm}^{3}\right.$, reference: $\left.0 / \mathrm{mm}^{3}\right)$ and rods $\left(1,080 / \mathrm{mm}^{3}\right.$, reference: 0 to $\left.300 / \mathrm{mm}^{3}\right)$, Normal neutrophil count $\left(2,640 / \mathrm{mm}^{3}\right.$, reference: 3,000 to $11,500 / \mathrm{mm}^{3}$ ) and lymphopenia (80/ $\mathrm{mm}^{3}$, reference: 1,000 to $4,800 / \mathrm{mm}^{3}$ ). The results of the biochemical tests performed were within normal limits. Leishmania sp. research was also performed by means of aspiration puncture by a fine needle of the lymph nodes and the presence of amastigotes forms in the analyzed material was not verified.

Thoracic radiography in the latero-lateral and ventrodorsal projections showed a marked unstructured interstitial pattern, suggesting infiltrative neoplastic process and increased cardiac silhouette, with ESR 10.9 (reference value: 10.5 ) and tracheal tract in the thoracic region displaced dorsally near the cardiac base, compatible with cardiomegaly. Abdominal ultrasound suggested nephropathy and inflammatory process in intestinal loops. The cytological examination performed by thin needle aspiration puncture of the nodules showed cellularity consisting of a predominance of individually arranged round cells, with moderate anisocytosis and cellular pleomorphism, cytoplasm of well-defined limits, of scarce to moderate amount and discrete basophilia, moderate anisocytosis and granular to dense chromatin, compatible with normal lymphoid tissue. Thus, 3 samples were collected from the nodules with the aid of a punch, two from the right dorsal lumbar region and one from the right pelvic limb for histopathological examination (Figure 1B). Malignant neoplasia was observed diffusely invading the deep dermis, the adipose panicle (Figure 2A) and the adjacent muscle tissue, characterized by round cells with large nuclei, some cleaved and evident nucleoli, scarce cytoplasm 
L.G. Rosseto, B.C.A. Pitol, P.A.T. Andreussi, et al. 2021. Non-Epitheliotropic Cutaneous Lymphoma with Systemic Dissemination in a Dog.

(Figure 2B) and numerous figures of mitosis, configuring the diagnosis of cutaneous lymphoma.

The material was submitted to immunohistochemical examination, immunoexpressing CD3 antibody ${ }^{1}$ and there was no marking of CD79 $\mathrm{a}^{1}, \mathrm{MUM}^{1}$, CD1a ${ }^{2}$, E-Cadherin ${ }^{3}$ and C-Kit ${ }^{3}$. The immunohistochemical and morphological profile favored the diagnosis of non-epitheliotropic T-lymphoma. After establishing the diagnosis of cutaneous lymphoma, the owner opted for euthanasia due to the compromised quality of life of the animal and unfavorable prognosis. In the necropsy examination it was compatible with multicentric lymphoma, involvement of lymph nodes, subcutaneous tissue, skeletal muscle, heart, pericardial sac, tongue and multifocal infiltrate of neoplastic cells where observed and there were $130 \mathrm{~mL}$ of free fluid in the chest, findings suggestive of multicentric lymphoma or infiltration by dissemination of cutaneous lymphoma.

\section{DISCUSSION}

There are important variations in the clinical behavior of cutaneous lymphoma, from an indolent course to a rapidly fatal systemic disease, as its diagnosis is made in most cases in advanced stages, with multifocal lesions or systemic dissemination [7]. However, few studies have shown the occurrence of primary skin lymphoma in other organs. There is a description of a case of cutaneous lymphoma with metastasis to the heart and kidneys with progression to paraneoplastic syndrome and death of the patient [1].

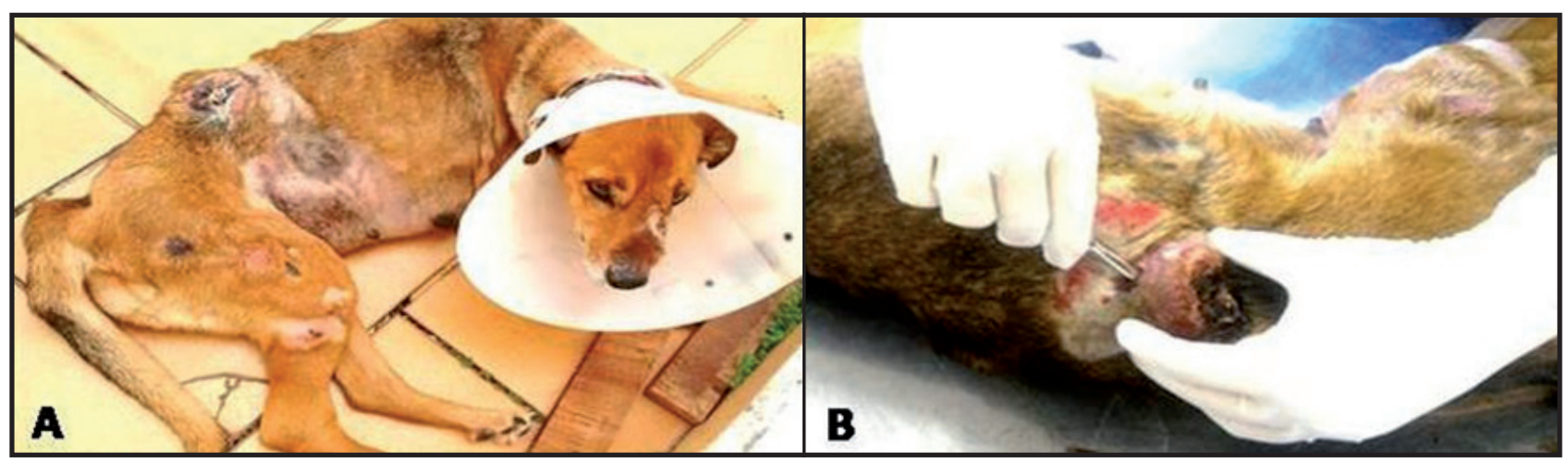

Figure 1. Dog with alopecic and non-pruritic subcutaneous nodules. A- Ulcerate nodules in multiple region and edema in right pelvic limb. B- Removal of a fragment from the dorsolumbar region using the punch for histopathological analysis.

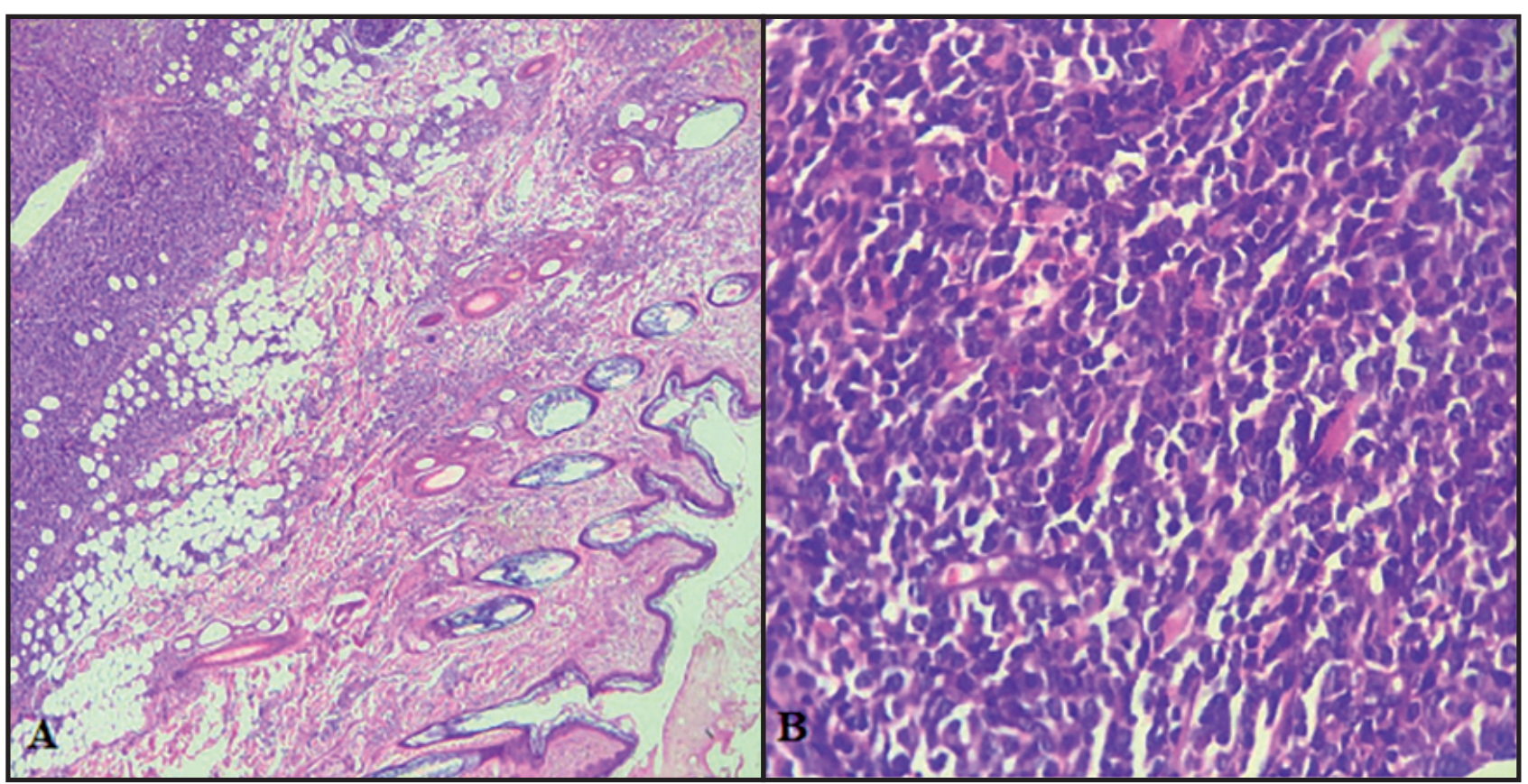

Figure 2. Skin fragments. A- Observe infiltration of round cell neoplasia in the dermis and panicle [10x]. B- Neoplasm of round cells with moderate pleomorphism, irregular nuclei and scarce cytoplasm [40x]. 
The majority of non-epitheliotropic skin lymphomas exhibit rapid progression and infiltration into lymph nodes and subsequent systemic involvement [2], which corroborates the evolution of the animal reported here. There is a report of a case of a 10-year-old dog with cutaneous lymphoma in oral mucosa and systemic dissemination of organs such as heart, lungs, liver, kidneys, spleen, among others [9].

The hematological alterations observed in the animal in this work have already been described by other authors. Regarding the hematological alterations, normocytic normochromic anemia is the most common alteration found in dogs with lymphoma, followed by leukocytosis, leukopenia, thrombocytopenia, neutrophilia and leukoerythroblastic reactions. Hematological alterations of patients with cancer may occur from the direct action of the tumor or paraneoplastic syndromes [4].

The pelvic limb edema observed in the patient probably occurred due to lymphatic fluid obstruction secondary to lymphadenomegaly. A study observed accumulation of extravascular fluid in 6 animals, four of them being edema of thoracic and pelvic limbs, thoracic effusion and ascites [5].

Immunophenotyping gained prognostic importance when it was found that dogs with T-cell lymphoma had a worse prognosis for life span and disease-free intervals than those with B-cell lymphoma [10]. The proliferation of $\mathrm{T}$ cells in canine epidermotropic lymphoma expresses CD3, a pan T-lymphocyte indication [5]. This immuno-marking pattern indicates that the neoplasm is derived from a line of $\mathrm{T}$ cells but does not differentiate between auxiliary (CD4+) and cytotoxic (CD8+) populations. A study showed studies with paraffin sections with antibodies for the specific manufacturer of CD3 T cells, B cells and markers of CD79a cells, E-cadherin and pan cytokeratin using routine avidin-biotin complex methods, evidencing neoplastic cells positive for CD3 and negative for CD79a, E-cadherin pan cytokeratin, thus demonstrating lineage tumors [3]. The histopathological and immunohistochemical examinations were decisive for the diagnosis of this report, because the expression CD3 and the negativity for CD79a proved that the neoplasia is of T lymphocyte lineage.

Cutaneous lymphoma is a malignant neoplasm with an unfavorable prognosis. The diagnosis of this disease is based on clinical-dermatological signs, fine needle cytology, histopathological and immunohistochemistry examination for definitive diagnosis. In this report, the definitive diagnosis was obtained by histopathology, but the immunohistochemistry determined the immunophenotype of the neoplasm as non-epitheliotropic T lymphocyte, being an important prognostic factor, because animals with this type of neoplasm have lower life expectancy.

\section{MANUFACTURERS}

${ }^{1}$ Dako. Santa Clara, CA, USA.

${ }^{2}$ Abdam. Cambridge, MA, USA.

${ }^{3}$ Cell Marque Corporation. Rocklin, CA, USA.

Acknowledgements. To VETPAT Laboratory of Veterinary Analysis by providing histopathology images.

Declaration of interest. The authors report no conflicts of interest. The authors alone are responsible for the content and writing of paper.

\section{REFERENCES}

1 Araújo C.M.T.D. 2017. Epitheliotropic lymphoma with heart and kidney metastasis in a dog: case report. Arquivo Brasileiro de Medicina Veterinária e Zootecnia. 69(6): 1461-1465. DOI: 10.1590/1678-4162-9291

2 Bosschere H. \& Declercq J. 2008. Cutaneous nonepitheliotropic B-cell lymphoma in a Golden retriever. Vlaams Diergeneeskundig Tijdschrift. 77: 315-318.

3 Bhang D.H., Choi U.S., Kim M.K., Choi E.H., Kang M.S., Hwang C.Y., Kim D.Y., Youn Y.H. \& Lee C.L. 2006. Epitheliotropic cutaneous lymphoma (Mycosis fungoides) in a dog. Journal Veterinary Science. 7(1): 97-99. DOI: $10.4142 /$ jvs.2006.7.1.97

4 Cápua M.L.B., Coleta F.E.D., Canesin A.P.M.N., Godoy A.V., Calazans S.G., Miotto M.R., Daleck C.R \& Santana A.E. 2011. Canine lymphoma: clinic, hematology and treatment with the Madison-Wisconsin protocol. Ciência Rural. 41(7): 1245-1251. DOI: 10.1590/S0103-84782011005000090

5 Cardoso M.J.L., Torres Neto R., Amorim R.L. \& Fabris V.E. 2006. Mycosis fungoides in a dog. Veterinária e Zootecnia. 13(20): 137-143. DOI: 10.35172/rvz.2006.v13.255

6 Daleck C.R., Nardi A.B. \& Calazans S.G. 2017. Lymphomas. In: Daleck C.R. \& Nardi A.B. (Eds). Oncologia em Cães e Gatos. 2.ed. Rio de Janeiro: Roca, pp.634-645. 
7 Duarte A.R. 2013. Lomustin skin lymphoma response - Clinical, immunohistochemical and MDR1 expression findings. 82f. Botucatu, SP. Dissertation (Masters in Veterinary Medicine) - Post-Graduate Program in Veterinary Medicine, State University Paulista Júlio Mesquita Filho.

8 Fontaine J., Bovens C. \& Bettenay S. 2009. Canine cutaneous epitheliotropic T-cell lymphoma: a review. Veterinary and Comparative Oncology. 7(1): 1-14. DOI: 10.1111/j.1476-5829.2008.00176

9 Mineshige M., Kawarai S., Yauchi T., Segawa K., Neo S., Sugahara G., Kamiie J., Hisasue M. \& Shirota K. 2016. Cutaneous epitheliotropic T-cell lymphoma with systemic dissemination in a dog. Journal of Veterinary Diagnostic Investigation. 28(3): 327-31. DOI: 10.1177/1040638716637642

10 Moreno K. 2008. Epidemiological, morphological and therapeutic aspects of cutaneous lymphoma and cell proliferation indices (PCNA E Ki-67) in multicenter lymphoma in dogs. 93f. Londrina, PR. Thesis (PhD in Veterinary Medicine) - Post-Graduate Program in Animal Science, State University of Londrina. 